\title{
Влияние особенностей зонного спектра на характеристики стимулированного излучения в узкозонных гетероструктурах с квантовыми ямами на основе $\mathrm{HgCdTe}$
}

\author{
(C) В.В. Румянцев ${ }^{1,2}$, Н.С. Куликов ${ }^{1,2}$, А.М. Кадыков ${ }^{1}$, М.А. Фадеев ${ }^{1}$, А.В. Иконников ${ }^{3}$, \\ А.С. Казаков ${ }^{3}$, М.С. Жолудев ${ }^{1}$, В.Я. Алешкин ${ }^{1,2}$, В.В. Уточкин ${ }^{1,2}$, Н.Н. Михайлов ${ }^{4,5}$, \\ C.А. Дворецкий ${ }^{4}$, С.В. Морозов ${ }^{1,2}$, В.И. Гавриленко ${ }^{1,2}$
}

\author{
${ }^{1}$ Институт ффизики микроструктур Российской академии наук, \\ 603950 Нижний Новгород, Россия \\ ${ }^{2}$ Нижегородской государственный университет им. Лобачевского, \\ 603950 Нижний Новгород, Россия \\ ${ }^{3}$ Московский государственный университет им. М.В. Ломоносова, \\ 119991 Москва, Россия \\ ${ }^{4}$ Институт фризики полупроводников Сибирского отделения Российской академии наук, \\ 630090 Новосибирск, Россия \\ ${ }^{5}$ Новосибирский государственный университет, \\ 630090 Новосибирск, Россия \\ E-mail: rumyantsev@ipm.sci-nnov.ru
}

(Получена 25 апреля 2018 г. Принята к печати 7 мая 2018 г.)

В квантовых ямах на основе $\mathrm{HgCdTe}$, помещенных в диэлектрический волновод из широкозонного твердого раствора $\mathrm{CdHgTe,} \mathrm{получено} \mathrm{стимулированное} \mathrm{излучение} \mathrm{в} \mathrm{диапазоне} \mathrm{длин} \mathrm{волн} \mathrm{20.3-17.4} \mathrm{мкм}$ на межзонных переходах при $T=8-50 \mathrm{~K}$. Гетероструктуры с квантовыми ямами на основе $\mathrm{HgCdTe}$ представляют интерес для создания длинноволновых лазеров на диапазон длин волн 25-60мкм, к настоящему времени недоступный для квантовых каскадных лазеров. Показано, что максимальная температура, до которой возможно получение стимулированного излучения, определяется положением „боковых“ максимумов на дисперсионных зависимостях в первой валентной подзоне квантовой ямы, и обсуждаются способы подавления безызлучательной рекомбинации в структурах с квантовыми ямами на основе $\mathrm{HgCdTe}$.

DOI: 10.21883/FTP.2018.11.46580.02

\section{1. Введение}

Проблема создания источников терагерцового (ТГц) излучения является одной из самых актуальных тем современной прикладной физики. Для многих приложений, связанных со спектроскопией, в качестве источников длинноволнового излучения выгодно использовать компактные полупроводниковые лазеры. Квантовые каскадные лазеры (ККЛ) демонстрируют высокие характеристики в спектральном диапазоне от 1 до 5 ТГц и выше 15 ТГц [1]. Однако для подавляющего большинства ККЛ используются полупроводники типа $\mathrm{A}^{\mathrm{III}} \mathrm{B}^{\mathrm{V}}(\mathrm{GaAs}$ или $\mathrm{InP}$ ), в которых фононное поглощение становится слишком сильным на частотах ниже 15 ТГц. ККЛ на основе GaN подступают к спектральному диапазону 5-15 ТГц со стороны низких частот (относительно частот оптических фононов), но их рабочие характеристики требуют значительного улучшения [2]. Межзонные лазеры представляются простой альтернативой ККЛ, но для их создания требуются узкозонные полупроводниковые структуры, в которых в свою очередь ожидается высокая эффективность безызлучательной оже-рекомбинации. Спектральный диапазон 5-15 ТГц к настоящему моменту частично перекрыт лишь диодными лазерами на основе халькогенидов свинца-олова, которые обеспечивают длины волн излучения вплоть до 46.5 мкм [3]. Фактор, который снижает эффективность оже-рекомбинации в $\mathrm{PbSnSe}(\mathrm{Te})$, - симметрия между законами дисперсии носителей в зоне проводимости и в валентной зоне $[4,5]$. Можно показать, что для выполнения законов сохранения энергии и импульса суммарная кинетическая энергия трех частиц, участвующих в процессе рекомбинации, должна быть выше некоторого порогового значения энергии $E_{\mathrm{th}}[6]$, которое зависит от энергетического спектра носителей. Для некоторых типов спектров носителей, например для релятивистских фермионов Дирака, выполнить законы сохранения энергии и импульса в ходе оже-процесса невозможно, т.е. $E_{\text {th }}$ бесконечна. Отметим, что симметрия законов дисперсии электронов и дырок реализуется для безмассовых фермионов Дирака в графене, однако вопрос об эффективности оже-рекомбинации в графеновых структурах до сих пор дебатируется, так что предельный случай релятивистского спектра с нулевой запрещенной зоной и линейным законом дисперсии требует отдельного рассмотрения [7]. Что же касается лазеров на основе халькогенидов свинца-олова, то их рабочие характеристики ограничены технологией роста: существуют труднопреодолимые проблемы в реализации квантовых ям (КЯ) для твердых растворов $\mathrm{PbSnSe}(\mathrm{Te})$, и остаточная концентрация носителей остается на высоком уровне $\sim 10^{17} \mathrm{~cm}^{-3}$.

Существуют альтернативные полупроводниковые системы, которые позволяют приблизиться к „графено- 
подобному“ закону дисперсии, но сохранить конечную ширину запрещенной зоны. Как было показано в многочисленных работах [8,9], одна из таких систем гетероструктуры с КЯ на основе $\mathrm{Hg}(\mathrm{Cd}) \mathrm{Te} / \mathrm{CdHgTe}$. В отличие от графена, в структурах на основе $\mathrm{HgCdTe}$ (КРТ) с КЯ можно перестраивать ширину запрещенной зоны путем изменения ширины КЯ и содержания $\mathrm{Cd}$ в ней. Современная молекулярно-лучевая эпитаксия (МЛЭ) обеспечивает высокое качество эпитаксиальных пленок КРТ не только на подложках $\mathrm{CdZnTe}$, но и на „альтернативных“ подложках GaAs [10]. Высокое качество эпитаксиальных структур $\mathrm{HgCdTe}$, выращенных на GaAs (013) подложках, было подтверждено в ходе исследований фотопроводимости $(Ф П)$ и фотолюминесценции (ФЛ) в среднем и дальнем инфракрасном диапазонах $(\lambda=15-30$ мкм $)[11,12]$. В КРТ структуpax с КЯ с шириной запрещенной зоны 60-80 мэВ наблюдалось увеличение времен жизни носителей с ростом интенсивности накачки вплоть до нескольких микросекунд благодаря насыщению центров рекомбинации Шокли-Рида-Холла [13]. На основе полученных результатов был сделан вывод, что оптическое усиление может быть получено при интенсивности накачки в пределах $10 \kappa В$ /см ${ }^{2}$ [14]. В недавней работе нами было получено стимулированное излучение (СИ) в КРТ структурах с КЯ на длине волны $\lambda=19.5$ мкм [15], в то время как ранее лазерная генерация в $\mathrm{HgCdTe}$ была продемонстрирована лишь в коротковолновой области среднего инфракрасного диапазона спектра (на длинах волн 2-5 мкм) [16,17].

Для структур, рассчитанных на генерацию длинноволнового излучения, требуется рост толстых эпитаксиальных слоев (общей толщиной до 20 мкм) для реализации диэлектрического волновода. При этом в рамках одного технологического цикла необходимо выполнить рост и толстых волноводных слоев толщиной несколько микрон, и массива узких КЯ со средней скоростью, соответствующей темпу роста объемных пленок $\mathrm{HgCdTe}$ (2-3 мкм/ч). Цель настоящей работы - исследование длинноволнового СИ из подобных структур и выявление факторов, определяющих наблюдаемые характеристики СИ, и возможных путей подавления безызлучательной рекомбинации в таких структурах. В работе [18] было продемонстрировано, что генерация СИ в таких структурах возможна, т. е. „быстрый“ рост структур не приводит к деградации их оптических свойств. Тем не менее профиль КЯ в волноводных структурах отличается от прямоугольного [19], что необходимо учитывать при анализе экспериментальных данных. В данной работе для модельного описания мы использовали профиль прямоугольной ямы с отличным от нуля содержанием кадмия, что позволяет хорошо описать зависимость ширины основного перехода от температуры, наблюдаемую в эксперименте.

\section{2. Методика эксперимента}

Исследуемые структуры были выращены методом МЛЭ на полуизолирующих GaAs (013) подложках с
ZnTe- и CdTe-буфером с in situ эллипсометрическим контролем состава и толщины слоев [11]. Ex situ характеризация структур проводилась с помощью измерений спектров межзонной ФП и ФЛ при различных температурах, из которых извлекалась температурная зависимость энергии ширины запрещенной зоны в КЯ. Сравнением этой зависимости с результатами расчетов в рамках модели Кейна $8 \times 8$ определялись ширина КЯ и содержание $\mathrm{Cd}$ в ней. Спектры ФП исследовались на образцах размером $5 \times 5$ мм в диапазоне температур 6-300 K с помощью фурье-спектрометра BrukerVertex70v со спектральным разрешением $8 \mathrm{~cm}^{-1}$. Образцы размещались в проточном криостате Oxford Instruments OptistatCF, который устанавливался в спектрометр так, чтобы образец находился в фокусе пучка излучения. В качестве источника излучения применялся глобар. Использовался светоделитель $\mathrm{KBr}$. В качестве фильтров применялись $\mathrm{ZnSe}$ и полиэтилен, что обеспечивало чувствительность установки в диапазоне 470-2800 $\mathrm{cm}^{-1}$, однако при этом в спектрах ФП имелись провалы в районе 1168, 1372 и $1452 \mathrm{~cm}^{-1}$, связанные с поглощением в полиэтилене. Наличие этих провалов учитывалось при оценке погрешности в определении красной границы ФП. Исследовались две структуры: структура 1 содержит 10 КЯ $\mathrm{Hg}_{0.87} \mathrm{Cd}_{0.13} \mathrm{Te} / \mathrm{Cd}_{0.65} \mathrm{Hg}_{0.35}$ Те толщиной 12 нм (что соответствует ширине запрещенной зоны 60 мэВ при $T=0$ ), структура 2 - $10 \mathrm{KЯ} \mathrm{Hg}_{0.87} \mathrm{Cd}_{0.13} \mathrm{Te} / \mathrm{Cd}_{0.65} \mathrm{Hg}_{0.35}$ Те толщиной $9.4 \mathrm{Hм}$ (что соответствует ширине запрещенной зоны 86 мэВ при $T=0)$.

Структуры не были преднамеренно легированы; остаточная концентрация носителей $p$-типа, полученная на основе холловских измерений, составляет порядка нескольких единиц $10^{10} \mathrm{~cm}^{-2}$, а типичная плотность дислокаций $\sim 10^{6} \mathrm{~cm}^{-2}$. Как было сказано выше, дизайн структур был ориентирован на эффективную локализацию света вблизи КЯ, для чего „активная“ область (10 КЯ) была выращена внутри толстых (толщина слоев составляет несколько микрон) волноводных слоев. Направление роста (013) не позволяет использовать сколотые грани образца в качестве зеркал „резонатора“, так как их плоскость составляет острый угол с плоскостью КЯ. Поэтому в данной работе исследовалось СИ, возникающее при однопроходовом усилении.

Исследования спектров ФЛ и СИ проводились в криостате замкнутого цикла с возможностью регулировки температуры в диапазоне $8-300 \mathrm{~K}$, оптически сопряженном с фурье-спектрометром Bruker Vertex 80v, работающим в режиме пошагового сканирования. Излучение выводилось через окно на основе КРС-5. В качестве приемника использовался кремниевый болометр, охлаждаемый жидким гелием. Для накачки структур использовались:

(i) титан-сапфировый лазер „Tsunami“ (длина волны 0.9 мкм, средняя мощность $400 \mathrm{MBT}$ ). Этот источник использовался при измерениях ФЛ с помощью модуляционной методики, описанной в работах $[11,20]$; 
(ii) лазерный комплекс Solar OPO с возможностью генерации излучения с длинами волн 1.94-2.3 мкм (блок КТР ОРО, энергия в импульсе до 5 мДж), длительностью импульса 10 нс и частотой повторения 10 Гц (за деталями эксперимента можно обратиться к работе [21]);

(iii) импульсный $\mathrm{CO}_{2}$-лазер (длина волны 10.6 мкм, длительность импульса $\sim 100$ нс с частотой повторения до 50 Гц, энергия в импульсе до 40 мДж).

Возбуждающее излучение во всех случаях полностью покрывало поверхность образца. Рассеянное излучение накачки с длиной волны 900-2300 нм отрезалось с помощью фильтра из арсенида индия или комбинации дихроичных фильтров. При накачке $\mathrm{CO}_{2}$-лазером в качестве фильтра использовалось входное окно спектрометра из полиметилпентена (ТРХ). Излучение собиралось с торца образца, так как направление, перпендикулярное оси роста структуры, оптимально для усиления [22]. При измерениях ФЛ использовалась конфигурация, при которой возбуждение осуществлялось под углом $45^{\circ}$ к оси роста структуры, а излучение собиралось с поверхности образца.

\section{3. Результаты и обсуждение}

На рис. 1 показаны спектры СИ структуры 1 при тем-

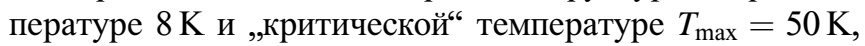
выше которой СИ не наблюдается. Ширина линии СИ на половине высоты составляет $\sim 1.5$ мэВ при небольшом превышении пороговой интенсивности оптического возбуждения, а с увеличением интенсивности накачки достигает $\sim 4$ мэВ, оставаясь тем не менее значительно уже, чем спектры ФЛ при непрерывном возбуждении, и гораздо уже, чем ширина типичной линии ФЛ от одной КЯ $\mathrm{HgCdTe}$ при импульсном возбуждении ( 10 мэВ) с той же интенсивностью [13]. Очевидно, что порог возникновения СИ растет с увеличением длины волны, а максимальная „рабочая“ температура $T_{\max }$ становится ниже. Чтобы понять энергетический „масштаб“, который определяет критическую температуру $T_{\max }$, рассмотрим энергетические спектры для двух КЯ с одной и той же шириной запрещенной зоны (равной ширине запрещенной зоны в структуре 1), представленные на рис. 2.

Для данных зонных структур были рассчитаны конфигурации возможных оже-процессов. Суммарная энергия носителей для процессов с участием двух дырок и электрона (CHНH), как при переходах дырки внутри одной подзоны, так и смене номера подзоны, превышает соответствующую энергию для процесса с участием двух электронов и дырки (ССНC), поэтому последний является наиболее важным при низкой температуре. Как видно из рис. 2, „критическая“ конфигурация и пороговая энергия $E_{\mathrm{th}}$ CСНС процесса определяются положением области „бокового“ максимума на дисперсионной зависимости дырок при больших значениях квазиимпульса. Для структуры $1 E_{\text {th }}=8$ мэВ, что при сравнении с температурой „гашения“ СИ $T_{\max }$, наблюдаемой в эксперименте, для структуры 1 дает $T_{\max } \approx E_{\mathrm{th}} / 2$.

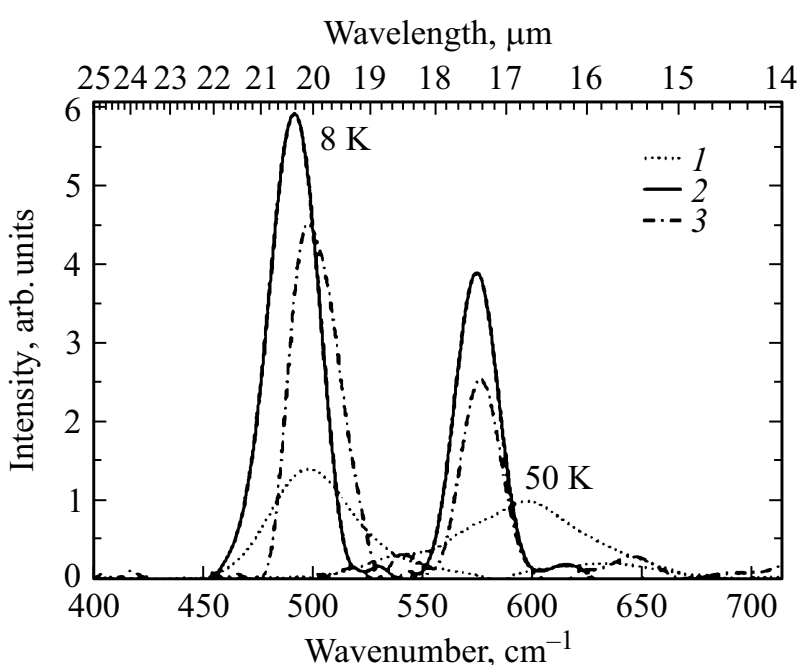

Рис. 1. Спектры ФЛ и СИ структуры 1 при $T=8$ и $50 \mathrm{~K}$ (амплитуды линий не нормированы): 1 - спектры ФЛ при возбуждении с длиной волны 900 нм и интенсивностью $10 \mathrm{~B}$ / $\left.\mathrm{cm}^{2}\right) ; 2$ - спектры СИ при накачке импульсами излучения параметрического генератора света с длиной волны 2300 нм и интенсивностью 10 кВт/см²; 3 - спектры СИ при накачке импульсами $\mathrm{CO}_{2}$-лазера с длиной волны 10.6 мкм и интенсивностью $20 \mathrm{\kappa ВT} / \mathrm{cm}^{2}(T=8 \mathrm{~K})$ и $60 \mathrm{\kappa BT} / \mathrm{cm}^{2}(T=50 \mathrm{~K})$.

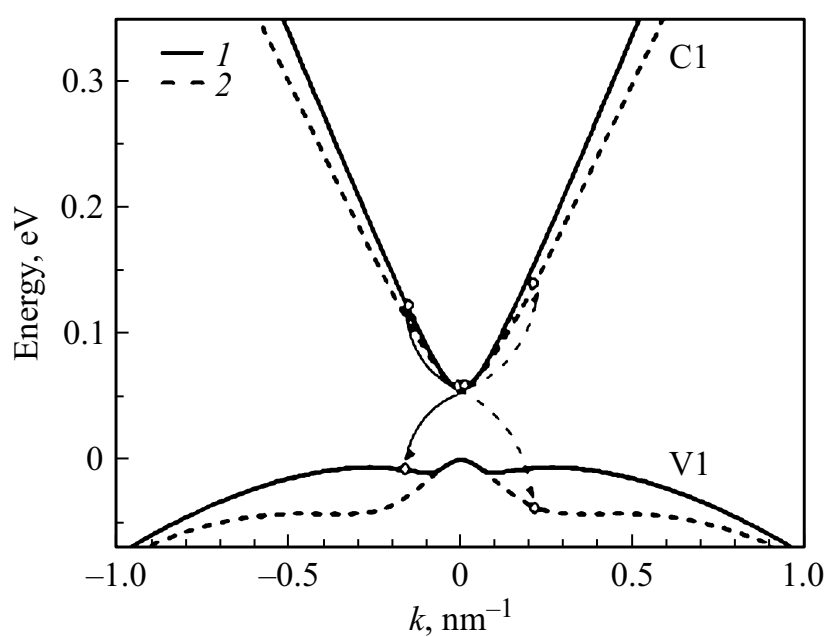

Pис. 2. Энергетический спектр (основные подзоны): 1 - КЯ $\mathrm{Hg}_{0.87} \mathrm{Cd}_{0.13} \mathrm{Te} / \mathrm{Cd}_{0.65} \mathrm{Hg}_{0.35} \mathrm{Te}$ толщиной 12 нм (структура 1), 2 - КЯ $\mathrm{HgTe} / \mathrm{Cd}_{0.65} \mathrm{Hg}_{0.35} \mathrm{Te}$ толщиной 4.2 нм при $T=0$. Стрелками схематически показаны переходы носителей для оже-процесса с участием 2 электронов и дырки с минимальной суммарной энергией.

Негативное влияние „боковых“ максимумов в валентной зоне на время жизни неравновесных носителей может быть уменьшено в КЯ из чистого НgТе. Как видно из рис. 2, для $\mathrm{HgTe} / \mathrm{CdHgTe}$ КЯ с той же шириной запрещенной зоны, что и в структуре 1 , боковые максимумы в валентной зоне смещаются в сторону меньших энергий, увеличивая пороговую энергию оже-процесса. Пороговая энергия для ССНС оже-процесса, рассчи- 


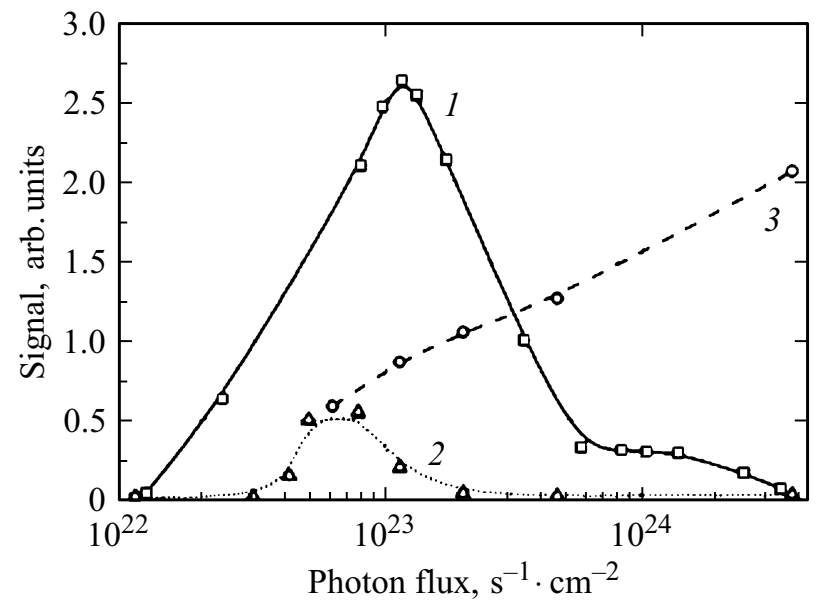

Рис. 3. Интегральная интенсивность СИ в зависимости от интенсивности накачки (эксперимент, выражена в количестве фотонов накачки на единицу площади в единицу времени) при $T=8 \mathrm{~K}: 1-$ в структуре 2 , длина волны СИ 14 мкм, при накачке импульсами излучения параметрического генератора света с длиной волны 2.3 мкм; 2 - в структуре 1 при накачке импульсами излучения параметрического генератора света с длиной волны 2.3 мкм; 3 - в структуре 1 при накачке импульсами $\mathrm{CO}_{2}$-лазера с длиной волны 10.6 мкм.

танная для такой структуры, составляет 45 мэВ, что позволяет оценить максимальную температуру, при которой возможно развитие СИ, путем экстраполяции как $T_{\max } \approx E_{\mathrm{th}} / 2 \sim 250 \mathrm{~K}$. Данная оценка, очевидно, является завышенной, так как с ростом температуры ширина запрещенной зоны растет быстрее, чем энергетическое расстояние между подзонами размерного квантования для дырок. Вследствие этого при высокой температуре станут возможны оже-процессы, связанные с забросом дырок из основной подзоны в возбужденные. Однако, как показывают расчеты, температура, при которой такие процессы становятся важны, для обсуждаемой структуры составляет около $100 \mathrm{~K}$, что позволяет рассчитывать на создание лазеров на длины волн свыше 20 мкм, работающие при температурах выше температуры кипения жидкого азота.

Помимо температуры кристаллической решетки, на эффективную температуру носителей влияет и их разогрев излучением накачки. При возбуждении структур излучением с длиной волны 2.3 мкм сильное влияние такого разогрева заметно уже в структурах с КЯ, рассчитанных на длины волн СИ около 14 мкм. На рис. 3 показаны измеренные зависимости интегральной интенсивности СИ от мощности накачки с длиной волны 2.3 мкм, иллюстрирующие „гашение“ СИ с ростом интенсивности накачки. Для „коротковолновой“ структуры 2 с шириной запрещенной зоны, соответствующей длине волны 14 мкм, интенсивность излучения нарастает с увеличением мощности накачки на порядок после порогового значения, а затем спадает, что, по-видимому, обусловлено „включением“ оже-рекомбинации вследствие роста температуры горячих носителей (см. [15]). В то же время для структуры 1 , рассчитанной на длины волн около 20 мкм, гашение наступает практически сразу после преодоления порога возникновения СИ. Переход к накачке с длиной волны 10.6 мкм резко уменьшает разогрев носителей, и интегральная интенсивность СИ монотонно растет с увеличением мощности накачки. Таким образом, эффект гашения СИ с ростом накачки, наблюдавшийся в [15], не является фундаментальным, а обусловлен использованием коротковолновой накачки.

\section{4. Заключение}

Таким образом, в данной работе продемонстрировано стимулированное излучение в узкозонных структурах с КЯ на основе $\mathrm{HgCdTe}$ в диапазоне длин волн от 20.3 до 17.4 мкм при перестройке температуры от 8 до $50 \mathrm{~K}$ соответственно. Показано, что максимальная температура, при которой возможна генерация СИ, определяется положением боковых максимумов в первой валентной подзоне. Использование узких ям $\mathrm{HgTe} / \mathrm{CdHgTe}$ и длинноволновой оптической накачки, при которой уменьшается эффект разогрева носителей, позволяет рассчитывать на генерацию СИ при температуре выше $77 \mathrm{~K}$, а также дальнейшее „продвижение“ в диапазон 20-60 мкм, где ККЛ к настоящему времени недоступны.

Работа выполнена при поддержке РФФИ (грант № 16-32-60172). Характеризация структур по измерениям спектров фотолюминесценции и расчеты зонного спектра структур проведены в рамках выполнения государственного задания ИФМ РАН, тема 0035-2014-0201, и при поддержке Министерства образования и науки (МК-4399.2018.2).

В работе использовано оборудование УСУ „Фемтоспектр“ ЦКП ИФМ РАН.

\section{Список литературы}

[1] M.S. Vitiello, G. Scalari, B. Williams, P. De Natale. Opt. Express, 23 (4), 5167 (2015).

[2] M.F. Anwar, T.W. Crowe, T. Manzur, W. Terashima, H. Hirayama. Proc. SPIE, 9483, 948304 (2015).

[3] K.V. Maremyanin, V.V. Rumyantsev, A.V. Ikonnikov, L.S. Bovkun, E.G. Chizhevskii, I.I. Zasavitskii, V.I. Gavrilenko. Semiconductors, 50 (12), 1669 (2016).

[4] J. Dimmock, I. Melngailis, A. Strauss. Phys. Rev. Lett., 16 (26), 1193 (1966).

[5] И.И. Засавицкий. Тр. ФИАН, 224, 3 (1993).

[6] V.N. Abakumov, V.I. Perel, I.N. Yassievich. Nonradiative Recombination in Semiconductors (North-Holland, Elsevier Science Publishers, 1991).

[7] G. Alymov, D. Svintsov, V. Vyurkov, V. Ryzhii, A. Satou. arXiv p. 1709.09015 (2018).

[8] B.A. Bernevig, T.L. Hughes, S.C. Zhang. Science, 314 (5806), 1757 (2006).

[9] A.M. Kadykov, J. Torres, S.S. Krishtopenko, C. Consejo, S. Ruffenach, M. Marcinkiewicz, D. But, W. Knap, S.V. Morozov, V.I. Gavrilenko, N.N. Mikhailov, S.A. Dvoretsky, F. Teppe. Appl. Phys. Lett., 108 (26), 262102 (2016). 
[10] V.S. Varavin, V.V. Vasiliev, S.A. Dvoretsky, N.N. Mikhailov, V.N. Ovsyuk, Yu.G. Sidorov, A.O. Suslyakov, M.V. Yakushev, A.L. Aseev. Opto-Electronics Rev., 11 (2), 99 (2003).

[11] S.V. Morozov, V.V. Rumyantsev, A.V. Antonov, K.V. Maremyanin, K.E. Kudryavtsev, L.V. Krasilnikova, N.N. Mikhailov, S.A. Dvoretskii, V.I. Gavrilenko. Appl. Phys. Lett., 104 (7), 072102 (2014)

[12] V.V. Rumyantsev, S.V. Morozov, A.V. Antonov, M.S. Zholudev, K.E. Kudryavtsev, V.I. Gavrilenko, S.A. Dvoretskii, N.N. Mikhailov. Semicond. Sci. Technol., 28 (12), 125007 (2013).

[13] S.V. Morozov, V.V. Rumyantsev, A.V. Antonov, A.M. Kadykov, K.V. Maremyanin, K.E. Kudryavtsev, N.N. Mikhailov, S.A. Dvoretskii, V.I. Gavrilenko. Appl. Phys. Lett., 105 (2), 022102 (2014).

[14] S. Ruffenach, A. Kadykov, V.V. Rumyantsev, J. Torres, D. Coquillat, D. But, S.S. Krishtopenko, C. Consejo, W. Knap, S. Winnerl, M. Helm, M.A. Fadeev, N.N. Mikhailov, S.A. Dvoretskii, V.I. Gavrilenko, S.V. Morozov, F. Teppe. APL Materials, 5 (3), 035503 (2017).

[15] S.V. Morozov, V.V. Rumyantsev, M.A. Fadeev, M.S. Zholudev, K.E. Kudryavtsev, A.V. Antonov, A.M. Kadykov, A.A. Dubinov, N.N. Mikhailov, S.A. Dvoretsky, V.I. Gavrilenko. Appl. Phys. Lett., 111 (19), 192101 (2017).

[16] J. Bleuse, J. Bonnet-Gamard, G. Mula, N. Magnea, P. JeanLouis. J. Cryst. Growth, 197 (3), 529 (1999).

[17] J.M. Arias, M. Zandian, R. Zucca, J. Singh. Semicond. Sci. Techn., 8 (1S), S255 (1993).

[18] В.В. Румянцев, А.М. Фадеев, А.А. Дубинов, В.В. Уточкин, Н.Н. Михайлов, С.А. Дворецкий, С.В. Морозов, В.И. Гавриленко. ФТП, 51 (12), 1616 (2017).

[19] Н.Н. Михайлов, С.А. Дворецкий, Д.Г. Икусов, В.Г. Ремесник, В.А. Швец, И.Н. Ужаков. Матер. XXII Междунар. симп. „Нанофизика и наноэлектроника“ (12-15 марта 2018, Нижний Новгород).

[20] J. Shao, W. Lu, X. Lü, F. Yue, Z. Li, S. Guo, J. Chu. Rev. Sci. Instrum., 77 (6), 063104 (2006).

[21] S.V. Morozov, V.V. Rumyantsev, A.M. Kadykov, A.A. Dubinov, K.E. Kudryavtsev, A.V. Antonov, N.N. Mikhailov, S.A. Dvoretskii, V.I. Gavrilenko. Appl. Phys. Lett., 108 (9), 092104 (2016)

[22] S.V. Morozov, V.V. Rumyantsev, A.A. Dubinov, A.V. Antonov, A.M. Kadykov, K.E. Kudryavtsev, D.I. Kuritsin, N.N. Mikhailov, S.A. Dvoretskii, V.I. Gavrilenko. Appl. Phys. Lett., 107 (4), 042105 (2015).

Редактор Г.А. Оганесян

\section{Effect of carrier spectrum features on stimulated emission characteristics in narrow-gap $\mathrm{HgCdTe}$ quantum waveguide structures}

V.V. Rumyantsev ${ }^{\mathbf{1}, 2}$, N.S. Kulikov ${ }^{\mathbf{1}, 2}$, A.M. Kadykov ${ }^{1}$, M. A. Fadeev' ${ }^{1}$, A.V. Ikonnikov ${ }^{3}$, A.S. Kazakov ${ }^{3}$, M.S. Zholudev ${ }^{1}$, V.Ya. Aleshkin ${ }^{1,2}$, V.V. Utochkin ${ }^{1,2}$, N.N. Mikhailov ${ }^{4,5}$, S.A. Dvoretskii ${ }^{4}$, V.I. Gavrilenko ${ }^{1,2}$, S.V. Morozov ${ }^{1,2}$

${ }^{1}$ Institute for Physics of Microstructures

Russian Academy of Sciences, 603950 Nizhny Novgorod, Russia

${ }^{2}$ Lobachevsky State University of Nizhny Novgorod, 603950 Nizhny Novgorod, Russia

${ }^{3}$ Lomonosov Moscow State University, 119991 Moscow, Russia

${ }^{4}$ Institute for Semiconductor Physics, Siberian Branch of Russian Academy of Sciences, 630090 Novosibirsk, Russia

${ }^{5}$ Novosibirsk State University, 630090 Novosibirsk, Russia

Abstract We report on stimulated emission in $20.3-17.4 \mu \mathrm{m}$ range from $\mathrm{HgCdTe}$ based QW structures, stacked inside the wide-gap $\mathrm{CdHgTe}$ dielectric waveguide, at $T=8-50 \mathrm{~K}$. $\mathrm{HgCdTe}$ structures are of interest for semiconductor lasers in the wavelength range of $25-60$ micron, which is not covered by quantum cascade lasers. We show that the maximum temperature, feasible for obtaining SE, is controlled by the position of side maxima of the dispersion law in the first hole subband. The promising routes to suppress the non-radiative recombination in $\mathrm{HgCdTe}$ structures are discussed. 